\title{
Effect of a strong magnetic field on the surface electric field of strange stars
}

\author{
X. P. Zheng and Y. W. Yu
}

The Institute of Astrophysics, Huazhong Normal University, Wuhan 430079, Hubei, PR China

e-mail: zhxp@phy.ccnu.edu.cn

Received 27 April 2005 / Accepted 26 July 2005

\section{ABSTRACT}

We made a detailed study of the properties of the electron layer near the quark surface of strange stars with strong $\left(\sim 10^{14}-10^{17} \mathrm{G}\right)$ magnetic fields. The electrostatic potential and the electric field at the quark surface were calculated as functions of the magnetic field intensity of bare strange stars. Using an ultrastrong $\left(B \geq 2.5 \times 10^{16} \mathrm{G}\right)$ magnetic field, we found that the distribution of electrons becomes an exponential function of radial distance, which is quite different in a magnetic field-free case. We also calculated the variation in gap width between the strange core and the normal nuclear crust for strange stars, which is due to magnetic field effect.

Key words. dense matter - stars: neutron - stars: magnetic fields

\section{Introduction}

Strange quark matter (SQM), made up of roughly equal numbers of up, down, and strange quarks, may be the absolute ground state of strong interaction. If this hypothesis is correct, strange quark stars (strange star, SS) may exist. Of course, it is very important to identify SSs. A new method for distinguishing SSs from neutron stars has been proposed recently based on the strong electric field at the quark surface of SSs (Xu et al. 2001; Usov 2001a).

Alcock et al. (1986) first pointed out that a very strong $\left(\sim 10^{17} \mathrm{~V} / \mathrm{cm}\right)$ electric field may exist near the bare quark surface. Due to the strong electric field, $\mathrm{e}^{ \pm}$pairs could be created spontaneously in a few empty quantum states; these pairs, which then are thermalized in the electron layer, subsequently annihilate into photons (Usov 1998, 2001b). This mechanism could play an important role in producing the thermal emission of bare SSs, because the SQM is a poor radiator of thermal photons at frequencies less than its plasma frequency $(\sim 20 \mathrm{MeV})$ (Alcock et al. 1986). Previous studies (Alcock et al. 1986; Kettner et al. 1995; Huang \& Lu 1997) have also shown that the strong electric field may be decisive for the existence of a nuclear crust on the quark surface of SSs, which may be created in a supernova or by accretion from the interstellar medium (Glendenning \& Weber 1992; Glendenning et al. 1995). This is due to the fact that the strong positive Coulomb barrier prevents atomic nuclei bound in the nuclear crust from coming into direct contact with the strange core. A SS with a nuclear crust may have different cooling time scales and surface properties from a bare SS. Thus the study of the nuclear crust in a SS is important for future observations of strange pulsars.

As pulsars, SSs are strongly magnetized. From a sample of more than 400 pulsars, the surface magnetic field intensity lies in the interval $\sim 2 \times 10^{10}-10^{13} \mathrm{G}$ (Manchester \& Tayor 1981). Several authors (Bisnovatyi-Kogan 1993; Duncan \& Thompson 1992; Thompson \& Duncan 1993) have proposed two different physical mechanisms that lead to an amplification of some initial magnetic fields in a collapsing star, fields as strong as $B \sim 10^{14}-10^{16} \mathrm{G}$, or even more. Considering the flux throughout the stars as a simple trapped primordial flux, the internal magnetic field may go up to $B \sim 10^{18}-10^{20} \mathrm{G}$. The energy of a charged particle changes significantly in the quantum limit if the magnetic field is equal to or greater than some critical value $B_{\mathrm{c}}=m_{i}^{2} c^{3} /\left(q_{i} \hbar\right) \mathrm{G}$, where $m_{i}$ and $q_{i}$ are the mass and charge, respectively. For $u$ and $d$ quarks of current mass $5 \mathrm{MeV}, B_{\mathrm{c}} \sim 4.4 \times 10^{15} \mathrm{G}$, while for $s$ quark of current mass $150 \mathrm{MeV}$, it is $\sim 10^{18} \mathrm{G}$. Especially for electrons of mass $0.5 \mathrm{MeV}$, a strong magnetic field that exceeds the critical value $B_{\mathrm{c}} \sim 4.4 \times 10^{13} \mathrm{G}$ forces the electron to behave as a one-dimensional gas rather than three-dimensional. Therefore, the quantum-mechanical effect of the magnetic field on the distribution of electrons near the quark surface of SSs cannot be neglected, just as the temperature effect leads to a considerable reduction of the electrostatic potential at the quark surface (Kettner et al. 1995). Phukon (2000) performed a perturbative expansion of the thermodynamics to the lowest order term in $B^{2} / \mu^{2}$ to reveal the magnetic effect. However, in his work, 
the details are lost in the middle magnetic field intensity, and the approach is invalid for the ultra-strong field case.

In this work we present a detailed investigation of the properties of the electron layer in the presence of a magnetic field and probe the magnetic field dependence of the gap width between the crust and the quark core. In the next section we describe the thermodynamic properties of bulk SQM in the presence of a strong magnetic field at zero temperature. In Sect. 3 we extend the Poisson equation near the quark surface to include the effect of magnetic field, and determine the electrostatic potential numerically. For an ultra-strong magnetic field, we simplify the equations and solve them analytically. In Sect. 4 we present the results of SSs with a thin crust. In the last section, we give our conclusions.

\section{Bulk SQM in strong magnetic fields}

For a constant magnetic field $B$, the single particle (species $i$ ) energy eigenvalue is written as

$\varepsilon_{i}=\sqrt{p_{i}^{2}+m_{i}^{2}+(2 n+s+1) q_{i} B}$,

where $n=0,1,2, \ldots$, are the principle quantum numbers for allowed Laudau levels, $s= \pm 1$ refers to $\operatorname{spin}$ up $(+)$ and down $(-)$, and $p_{i}$ and $q_{i}$ are the components of particle momentum along the field direction and the absolute value of the electric charge, respectively. Setting $2 n+s+1=2 v$, where $v=0,1,2, \ldots$, we can rewrite the single particle energy eigenvalue in the following form

$\varepsilon_{i}=\sqrt{p_{i}^{2}+m_{i}^{2}+2 v q_{i} B}$

Now it is very easy to show that the $v=0$ state is singly degenerate, while the others with $v \neq 0$ are doubly degenerate. To a zero temperature approximation, the thermodynamic potential of the $i$ th species is given by (Chakrabarty \& Sahu 1996)

$$
\begin{aligned}
\Omega_{i}= & -\frac{g_{i} q_{i} B}{4 \pi^{2}} \sum_{v_{i}=0}^{v_{i, m}} b_{v_{i} 0}\left\{\mu_{i} \sqrt{\mu_{i}^{2}-m_{i}^{2}-2 v_{i} q_{i} B}\right. \\
& \left.-\left(m_{i}^{2}+2 v_{i} q_{i} B\right) \ln \left[\frac{\mu_{i}+\sqrt{\mu_{i}^{2}-m_{i}^{2}-2 v_{i} q_{i} B}}{\sqrt{m_{i}^{2}+2 v_{i} q_{i} B}}\right]\right\}
\end{aligned}
$$

where $g_{i}$ and $\mu_{i}$ are the degeneracy (equaling 6 for quarks and 2 for electron) and chemical potential of the $i$ th species, $b_{v_{i} 0}=1-\frac{1}{2} \delta_{v_{i} 0}$. The upper limit $v_{i, m}$ is the greatest integer not exceeding $\left(\mu_{i}^{2}-m_{i}^{2}\right) / 2 q_{i} B$.

For a SS, the net positive charge of the quarks will be balanced locally by electrons up to radial distances $r \leq R_{m}$, where $R_{m}$ is only slightly smaller than the stellar radius $R$. This charge neutrality condition of bulk matter is expressed as

$2 n_{u}-n_{d}-n_{s}-3 n_{e}=0$.

And the baryon number density of the system is given by

$n_{\mathrm{B}}=\frac{1}{3}\left(n_{d}+n_{u}+n_{s}\right)$ which is considered as a constant parameter. For bulk SQM, assuming the condition of $\beta$ equilibrium, we have

$\mu_{d}=\mu_{s}=\mu$,

$\mu_{u}=\mu-\mu_{e}$

To solve the above equations for a given magnetic field and baryon number density, we need the relationship between $n_{i}$ and $\mu_{i}$. From the well-known thermodynamic relation, the expression for the number density of the $i$ th species $(i=u, d$ and $e$ ) is given by (Chakrabarty \& Sahu 1996)

$n_{i}=-\left(\frac{\partial \Omega_{i}}{\partial \mu_{i}}\right)=\frac{g_{i} q_{i} B}{2 \pi^{2}} \sum_{v_{i}=0}^{v_{i, m}} b_{v_{i} 0} \sqrt{\mu_{i}^{2}-m_{i}^{2}-2 v_{i} q_{i} B}$.

To the weak magnetic field limit $\left(\mu_{i}^{2}-m_{i}^{2}\right) / 2 q_{i} B \rightarrow \infty$, taking $\sum_{v_{i}=0}^{v_{i, m}} \rightarrow \int_{0}^{v_{i, m}} \mathrm{~d} v_{i}$, we can obtain the classical expression of the number density

$n_{i}=\frac{g_{i}\left(\mu_{i}^{2}-m_{i}^{2}\right)^{3 / 2}}{6 \pi^{2}}$,

which is valid for $s$ quark due to its large mass

$n_{s}=\frac{\left(\mu_{s}^{2}-m_{s}^{2}\right)^{3 / 2}}{\pi^{2}}$

On the other hand, for an ultra-strong field $B$ that satisfies $\left(\mu_{i}^{2}-m_{i}^{2}\right) / 2 q_{i} B<1$, only the state $v_{i}=0$ is allowed. We then can get rid of the sum for Laudau levels in Eq. (8) to read

$n_{i}=\frac{g_{i} q_{i} B}{4 \pi^{2}} \sqrt{\mu_{i}^{2}-m_{i}^{2}}$.

Now we can solve Eqs. (4)-(7) to obtain the number densities and the chemical potential of all species in the interior including the vicinity of $R_{m}\left(r \leq R_{m}\right)$ of SSs with various values of $B$.

\section{Bare quark surface}

\subsection{Equations and numerical results}

Beyond $R_{m}$, in the region $R_{m} \leq r \leq \infty$, the charge neutrality is a global rather than a local condition. A concise model for this layer was first developed by Alcock et al. (1986). In a simple Thomas-Fermi model, the electrostatic potential near the quark surface is described by Poisson's equation

$\frac{\mathrm{d}^{2} V}{\mathrm{~d} r^{2}}= \begin{cases}4 \pi e^{2}\left(n_{e}-n_{q}\right), & r \leq R, \\ 4 \pi e^{2} n_{e}, & r>R,\end{cases}$

where $V / e$ is the electrostatic potential. The chemical equilibrium of the electrons in the layer implies that the value $\mu_{\infty}=\mu_{e}-V$ is constant. Since far outside the star, both $V$ and $\mu_{e}$ tend to 0 , it follows that $\mu_{\infty}=0$ and $\mu_{e}=V$, and

$n_{q}=\frac{2}{3} n_{u}-\frac{1}{3} n_{d}-\frac{1}{3} n_{s}$

is the quark charge density inside the quark surface. Because $R$ and $R_{m}$ differ only by a few hundred fermi, density $n_{q}$ in this 
range can be treated as being independent of $r$. Because of the local electric charge neutrality inside $R_{m}$,

$n_{q}=n_{e}\left(R_{m}\right)=\frac{g_{e} e B}{2 \pi^{2}} \sum_{v=0}^{v_{m}} b_{v 0} \sqrt{V_{q}^{2}-m_{e}^{2}-2 v e B}$,

where $V_{q}$ is clearly the chemical potential corresponding to the net charge density of quarks inside $R_{m}$, which can be obtained from Eqs. (4)-(7).

Assuming the magnetic field in the electron layer is uniform and substituting Eq. (8) into Eq. (12), we come to

$$
\frac{\mathrm{d}^{2} V}{\mathrm{~d} r^{2}}=\left\{\begin{aligned}
\frac{2 g_{e} e^{3} B}{\pi}\left(\sum_{v=0}^{v_{m}} b_{\nu 0} \sqrt{V^{2}-m_{e}^{2}-2 v e B}\right. & \\
\left.-\sum_{v^{\prime}=0}^{v_{m}^{\prime}} b_{v^{\prime} 0} \sqrt{V_{q}^{2}-m_{e}^{2}-2 v^{\prime} e B}\right), & r \leq R, \\
\frac{2 g_{e} e^{3} B}{\pi} \sum_{v=0}^{v_{m}} b_{v 0} \sqrt{V^{2}-m_{e}^{2}-2 v e B}, & r>R .
\end{aligned}\right.
$$

The boundary conditions for the above equation are $V \rightarrow V_{q}$ and $\mathrm{d} V / \mathrm{d} r \rightarrow 0$ as $r \rightarrow 0$, and $V \rightarrow 0$ and $\mathrm{d} V / \mathrm{d} r \rightarrow 0$ as $r \rightarrow \infty$, respectively. By integrating Eq. (15) we can obtain

$$
\frac{\mathrm{d} V}{\mathrm{~d} r}=\left\{\begin{array}{c}
-\sqrt{\frac{2 g_{e} e^{3} B}{\pi}}\left\{\sum _ { v = 0 } ^ { v _ { m } } b _ { v 0 } \left[V \sqrt{V^{2}-m_{e}^{2}-2 v e B}\right.\right. \\
-V_{q} \sqrt{V_{q}^{2}-m_{e}^{2}-2 v e B} \\
\left.-\left(m_{e}^{2}+2 v e B\right) \ln \left(\frac{V+\sqrt{V^{2}-m_{e}^{2}-2 v e B}}{V_{q}+\sqrt{V_{q}^{2}-m_{e}^{2}-2 v e B}}\right)\right] \\
\left.-2 \sum_{v^{\prime}=0}^{v_{m}^{\prime}} b_{\nu^{\prime} 0}\left(V-V_{q}\right) \sqrt{V_{q}^{2}-m_{e}^{2}-2 v^{\prime} e B}\right\}^{\frac{1}{2}}, \\
-\sqrt{\frac{2 g_{e} e^{3} B}{\pi}}\left\{\sum _ { v = 0 } ^ { v _ { m } } b _ { v 0 } \left[V \sqrt{V^{2}-m_{e}^{2}-2 v e B},\right.\right. \\
\left.\left.-\left(m_{e}^{2}+2 v e B\right) \ln \left(\frac{V+\sqrt{V^{2}-m_{e}^{2}-2 v e B}}{\sqrt{m_{e}^{2}+2 v e B}}\right)\right]\right\}^{\frac{1}{2}}, \\
r>R .
\end{array}\right.
$$

The global charge neutrality determines the potential at the quark surface to be (Kettner et al. 1995)

$V(R)=V_{q}-\frac{P_{e}\left(R_{m}\right)-P_{e}(\infty)}{n_{q}}$,

where $P_{e}(R)$ is the kinetic pressure of electrons, which could be obtained by the thermodynamic relation $P_{e}=-\Omega_{e}$. In Fig. 1 we show the variation of $V_{q}$ and $V(R)$ with the magnetic field intensity $B$. We can see $V_{q}$ is nearly independent of $B$ for our range of fields $\left(\sim 10^{14}-10^{17} \mathrm{G}\right)$. And the relation $V(R) \simeq \frac{3}{4} V_{q}$ applied widely in previous studies is a good approximation for a weak $\left(B<10^{15} \mathrm{G}\right)$ magnetic field, while $V(R) \simeq \frac{1}{2} V_{q}$ for an ultra-strong $\left(B>2.5 \times 10^{16} \mathrm{G}\right)$ field. In the middle range $\left(B \sim 10^{15}-2.5 \times 10^{16} \mathrm{G}\right), V(R)$ oscillates with magnetic field intensity. These oscillations are easily understood in accordance with Laudau levels. As is known, the maximum level $v_{m}$, the integer of the ratio $\frac{\left(\mu_{e}-m_{e}\right)^{2}}{2 e B}$, takes separate values as a function of $B$. We also show the variation of $v_{m}$ with $B$ in Fig. 1 . It is obvious that the oscillation of $V(R)$ occurs synchronously following the skip of $v_{m}$. For a weak field, $v_{m}$ has a very large value as $\frac{\Delta v}{v_{m}} \rightarrow 0$ so that the oscillation amplitude is very small.

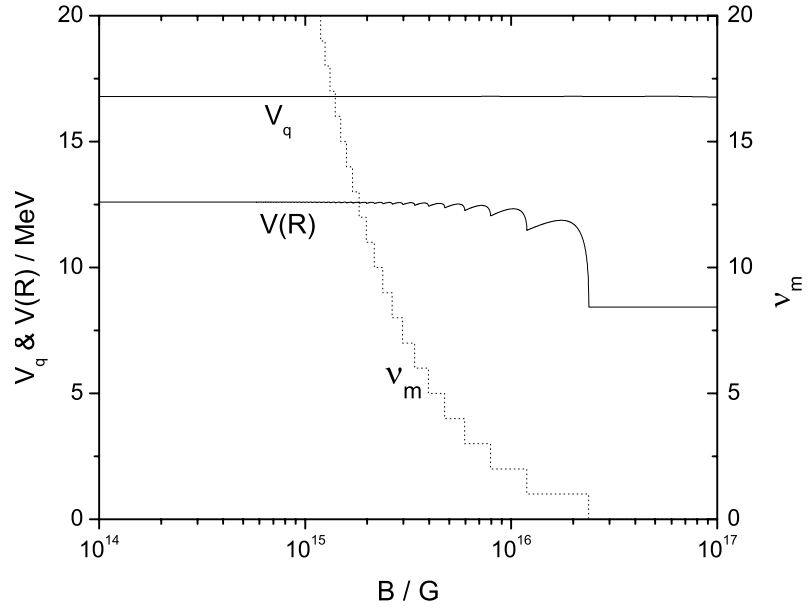

Fig. 1. $V_{q}$ and $V(R)$ vs. magnetic field intensity $B$ for $n_{\mathrm{B}}=2.5 n_{0}$. The dotted curve shows the number of the maximum Laudau level $v_{m}$ with different $B$.

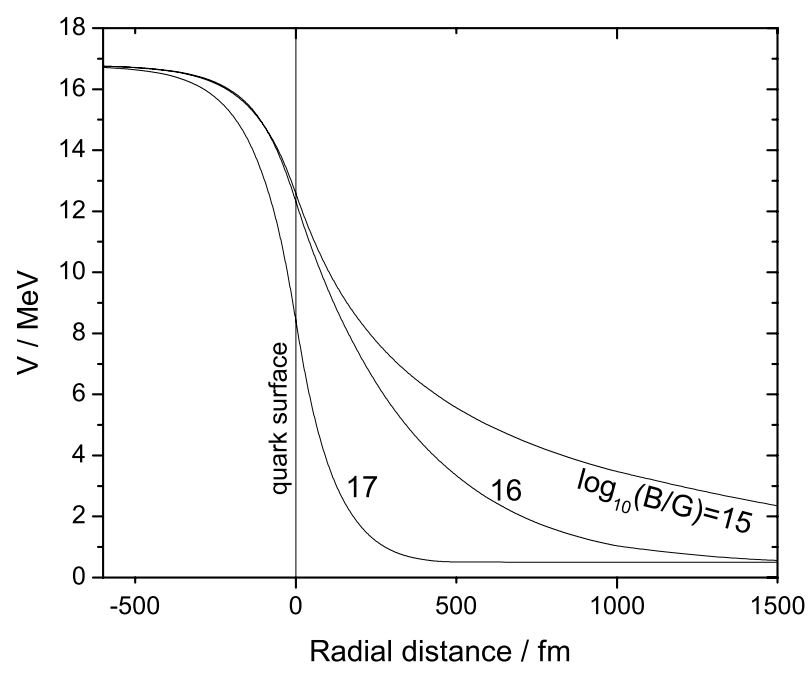

Fig. 2. Electrostatic potential of electrons in the close vicinity inside and outside of the quark surface of Bare SSs. The location of the surface is indicated by the vertical line. The figures assigned to these curves refer to magnetic field intensity.

However, the quantum effect becomes important due to the low value of $v_{m}$ that has the comparable magnitude of $\Delta v$ in the middle field intensity. Therefore $V(R)$ oscillates considerably in this range depending on the separate values of $v_{m}$. The oscillation disappears until $v_{m}$ vanishes for very strong magnetic fields. Furthermore, not only $V(R)$, but also other quantities involving the summation of Laudau levels show oscillations (see subsequent figures).

The behaviors of $V(r)$ that vary with respect to $B$ are exhibited in Fig. 2. We can see the stronger the magnetic field is, the larger the potential changes with $r$. To illustrate the details, we plot the curve of the electric field (at the quark surface $\left.E(R)=(-\mathrm{d} V / \mathrm{d} r)_{r=R}\right)$ versus magnetic field in Fig. 3. With the increase in $B$, the electric field increases remarkably after oscillations within a range $\left(\sim 10^{15}-2.5 \times 10^{16} \mathrm{G}\right)$. 


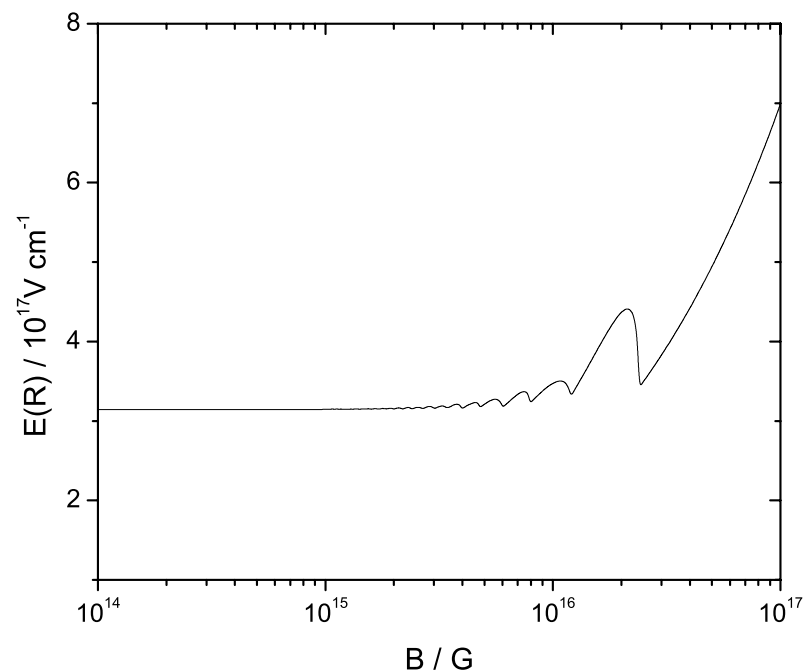

Fig. 3. Electric field at the quark surface vs. magnetic field intensity $B$.

\subsection{Ultra-strong magnetic field approximation}

At ultra-strong magnetic field $\left(B \geq 2.5 \times 10^{16} \mathrm{G}\right.$ for $V_{q} \sim$ $17 \mathrm{MeV}$ ), only the lowest Laudau level is allowed, and Eq. (16) becomes to

$$
\frac{\mathrm{d} V}{\mathrm{~d} r}=\left\{\begin{array}{l}
-\sqrt{\frac{2 e^{3} B}{\pi}} \times \\
{\left[V \sqrt{V^{2}-m_{e}^{2}}-\left(2 V-V_{q}\right) \sqrt{V_{q}^{2}-m_{e}^{2}}\right.} \\
\left.-m_{e}^{2} \ln \left(\frac{V+\sqrt{V^{2}-m_{e}^{2}}}{V_{q}+\sqrt{V_{q}^{2}-m_{e}^{2}}}\right)\right]^{\frac{1}{2}}, \quad r \leq R, \\
-\sqrt{\frac{2 e^{3} B}{\pi} \times} \times \\
{\left[V \sqrt{V^{2}-m_{e}^{2}}-m_{e}^{2} \ln \left(\frac{V+\sqrt{V^{2}-m_{e}^{2}}}{m_{e}}\right)\right]_{r}^{\frac{1}{2}} .} \\
\end{array}\right.
$$

Since $V \gg m_{e}$, the above equation can be written more simply, if we ignore the mass of the electron, to read

$$
\frac{\mathrm{d} V}{\mathrm{~d} r}=\left\{\begin{array}{cc}
-\sqrt{\frac{2 e^{3} B}{\pi}}\left(V_{q}-V\right), & r \leq R, \\
-\sqrt{\frac{2 e^{3} B}{\pi}} V, & r>R .
\end{array}\right.
$$

By integrating this equation and using the relation $V(R) \simeq \frac{1}{2} V_{q}$ (obtained from Eq. (17) for ultra-strong field approximation), we get the expression of $V$, and $E=-\mathrm{d} V / \mathrm{d} r$ as a function of radial distance $r$

$$
\begin{gathered}
V(r)=\left\{\begin{array}{c}
V_{q}\left\{1-\frac{1}{2} \exp \left[\sqrt{\frac{2 e^{3} B}{\pi}}(r-R)\right]\right\}, \quad r \leq R, \\
\frac{1}{2} V_{q} \exp \left[-\sqrt{\frac{2 e^{3} B}{\pi}}(r-R)\right], \quad r>R .
\end{array}\right. \\
E(r)=\left\{\begin{array}{cc}
\frac{1}{2} \sqrt{\frac{2 e^{3} B}{\pi}} V_{q} \exp \left[\sqrt{\frac{2 e^{3} B}{\pi}}(r-R)\right], \quad r \leq R, \\
\frac{1}{2} \sqrt{\frac{2 e^{3} B}{\pi}} V_{q} \exp \left[-\sqrt{\frac{2 e^{3} B}{\pi}}(r-R)\right], \quad r>R .
\end{array}\right.
\end{gathered}
$$

Substituting Eq. (20) into Eq. (11) in a zero electron mass approximation, we get the number density of the electron

$$
n_{e}(r)=\left\{\begin{array}{c}
\frac{e B}{2 \pi^{2}} V_{q}\left\{1-\frac{1}{2} \exp \left[\sqrt{\frac{2 e^{3} B}{\pi}}(r-R)\right]\right\}, r \leq R, \\
\frac{e B}{4 \pi^{2}} V_{q} \exp \left[-\sqrt{\frac{2 e^{3} B}{\pi}}(r-R)\right], \quad r>R .
\end{array}\right.
$$

Equations (20)-(22) show that the electrostatic potential, electric field, and electron number density are all exponential functions of radial distance. They are quite different from the classical power-law expressions (Kettner et al. 1995).

\section{Thin crust}

The large outwardly directed electric field is capable of supporting some normal material, which then gives birth to a thin crust. Two regions should be distinguished just outside the quark surface. The first one extends from the surface at $r=R$ to the radial distance where the inner nuclear crust (the crust's base) begins, denoted $r=R_{\mathrm{c}}$. The associated width, $\Delta R \equiv R_{\mathrm{c}}-R$, is referred to henceforth as the gap. The second region begins at $R_{\mathrm{c}}$ and extends in the radial outward direction toward infinity. Then the corresponding Poisson equation reads

$\frac{\mathrm{d}^{2} V}{\mathrm{~d} r^{2}}=\left\{\begin{array}{c}4 \pi e^{2}\left(n_{e}-n_{q}\right), \quad r \leq R, \\ 4 \pi e^{2} n_{e}, \quad R<r<R_{\mathrm{c}}, \\ 4 \pi e^{2}\left(n_{e}-n_{\text {ion }}\right), \quad R_{\mathrm{c}} \leq r,\end{array}\right.$

where $n_{\text {ion }}$ is the positive charge density of ions. For simplicity and convenience, we assume the electrostatic potential in the nuclear crust regime $\left(r \geq R_{\mathrm{c}}\right)$ is constant, as several authors (Alcock et al. 1986; Kettner et al. 1995) have done, i.e., $V\left(r \geq R_{\mathrm{c}}\right) \equiv V_{\mathrm{c}}$ (= const.). Then the boundary conditions become $V \rightarrow V_{q}$ as $r \rightarrow R_{m}$ and $V \rightarrow V_{\mathrm{c}}$ as $r \rightarrow R_{\mathrm{c}}$.

Since the mass of ions in the crust is too large for our range of magnetic field, the lattice and positive charge density of ions should not be influenced by magnetic field. For a crust, we define a symbolic electrostatic potential $V_{\mathrm{c}}^{*}$ as

$$
\frac{1}{3 \pi^{2}}\left(V_{\mathrm{c}}^{* 2}-m_{e}^{2}\right)^{3 / 2} \equiv \frac{g_{e} e B}{2 \pi^{2}} \sum_{\nu=0}^{v_{m}} b_{\nu 0} \sqrt{V_{\mathrm{c}}^{2}-m_{e}^{2}-2 v e B} .
$$

This new symbol denotes the effective potential at the base of the crust in the absence of a magnetic field. We use it to designate different crusts.

The numerical results of $V_{q}, V(R)$, and $V_{\mathrm{c}}$ (for $V_{\mathrm{c}}^{*}=$ $10 \mathrm{MeV})$ are shown in Fig. 4. The conventional equation $V(R)=\frac{3}{4} V_{q}+\frac{1}{4} \frac{V_{\mathrm{c}}^{4}}{V_{q}^{3}}$ is also correct for weak magnetic fields $\left(B<10^{15} \mathrm{G}\right)$, while $V(R)=\frac{1}{2} V_{q}+\frac{1}{2} \frac{V_{\mathrm{c}}^{2}}{V_{q}}$ for ultra-strong magnetic field approximation. Using this numerical result, we show the results of calculations of the electrostatic potential with respect to different magnetic fields for $V_{\mathrm{c}}^{*}=10 \mathrm{MeV}$ in Fig. 5 . In previous studies (Alcock et al. 1986; Kettner et al. 1995; Huang \& Lu 1997), a minimum value of 200 fm is established as the lower bound on gap width necessary to guarantee the crust against strong interaction with the star's strange core. Figure 6 shows the curves of gap width versus magnetic field intensity for several crusts. If we adopt $200 \mathrm{fm}$ as a criterion for gap width, then the maximum value of $V_{\mathrm{c}}^{*}$ may be $9 \mathrm{MeV}$ : the corresponding crust could be stable for all magnetic fields in our range $\left(B<10^{17} \mathrm{G}\right)$. Our results demonstrate that the gap width of various crusts converges to a narrow region $(\sim 200-400 \mathrm{fm})$ with the increase in $B$. For lighter crusts, the width decreases considerably, i.e. from $\Delta R=1556 \mathrm{fm}$ at $B=10^{15} \mathrm{G}$ to $\Delta R=387 \mathrm{fm}$ at $B=10^{17} \mathrm{G}$ for $V_{\mathrm{c}}^{*}=3 \mathrm{MeV}$. 


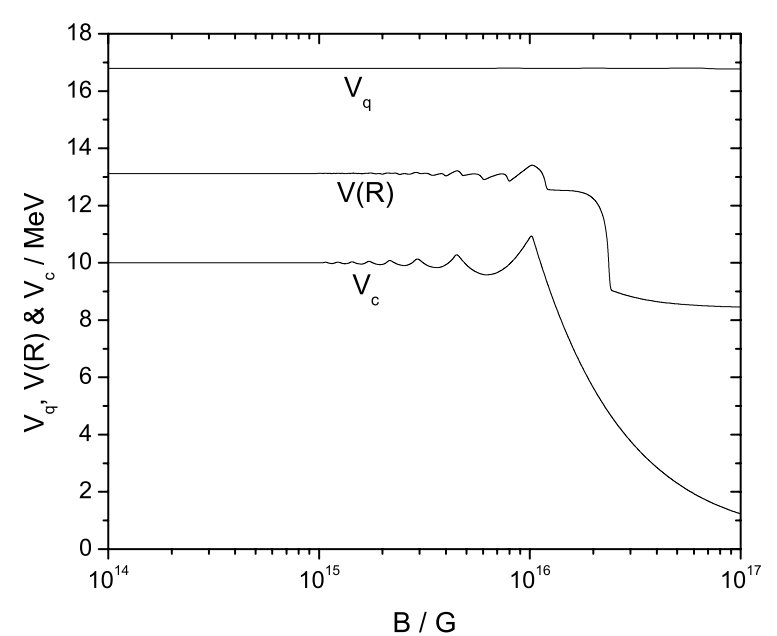

Fig. 4. $V_{q}, V(R)$ and $V_{\mathrm{c}}$ vs. the magnetic field intensity $B$. A representative value for symbolic electrostatic potential $V_{\mathrm{c}}^{*}=10 \mathrm{MeV}$.

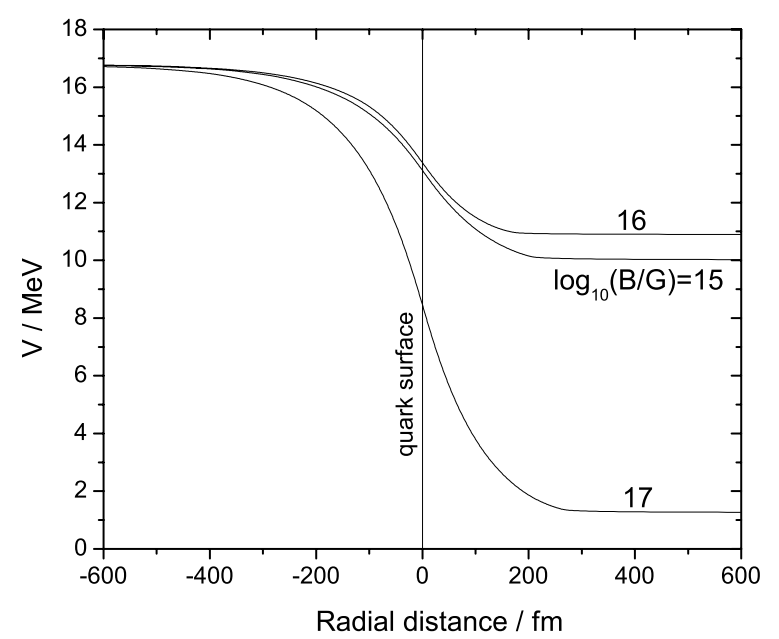

Fig. 5. Same as Fig. 2, but for SSs with a crust with $V_{\mathrm{c}}^{*}=10 \mathrm{MeV}$.

However, a realistic gap width should be influenced by the mechanical balance condition (Huang \& Lu 1997). This condition requires the electron layer above the quark surface to partially penetrate the crust. The gap width can be over $200 \mathrm{fm}$ for only the very lighter crusts when the penetrated part is deducted from our present widths. The details will be studied in our future work.

\section{Conclusions}

In this paper, the properties of the electric field near the surface of strange stars with magnetic field are studied thoroughly. We find that a weak $\left(B \leq 10^{15} \mathrm{G}\right)$ magnetic field has hardly any effect on the surface electric field of bare SSs. Secondly, in the middle range $\left(B \sim 10^{15}-2.5 \times 10^{16} \mathrm{G}\right)$ of magnetic field intensity, both the electrostatic potential and electric field have an oscillation as $B$ changes. Thirdly, for ultra-strong $\left(B \geq 2.5 \times 10^{16} \mathrm{G}\right)$ magnetic fields, the electrostatic potential at quark surface reduces considerably, accompanied by an

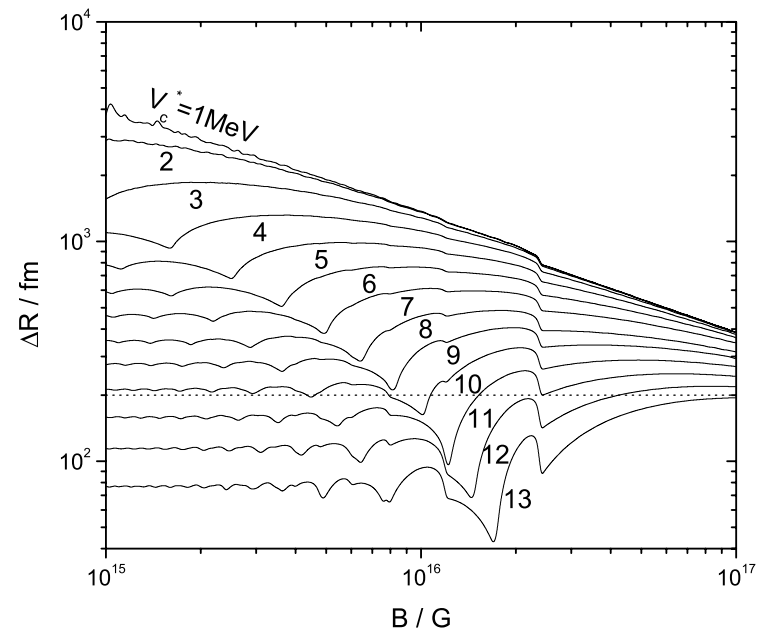

Fig. 6. Gap width vs. magnetic field intensity. The labels refer to symbolic electrostatic potential (in $\mathrm{MeV}$ ).

increase in the electric field. The electrostatic potential, the electric field, and the electron number density are all exponential functions of radial distance, which is quite different from the field-free case.

We took the effect of magnetic field on the nuclear crust into account. With the increase in magnetic field intensity, the gap width of various crusts converges to a narrow region ( 200-400 fm). The lighter crusts have a quick decrease in gap width, while the heavier ones suffer a slight increase in gap as $B$ is strengthened. We infer that those may influence the mass of the crust.

Acknowledgements. We would like to thank Dr. Y. F. Huang for the useful discussion and to acknowledge the support by NFSC under Grant Nos 10373007, 90303007, and the Ministry of Education of China with project No. 704035 .

\section{References}

Alcock, C., Farhi, E., \& Olinto, A. 1986, ApJ, 310, 261

Bisnovatyi-Kogan, G. S. 1993, Astron. Astrophys. Trans., 3, 287

Chakrabarty, S., \& Sahu, P. K. 1996, Phys. Rev. D, 53, 4687

Duncan, R. C., \& Thompson, C. 1992, ApJ, 392, L9

Glendenning, N. K., \& Weber, F. 1992, ApJ, 400, 647

Glendenning, N. K., Kettner, Ch., \& Weber, F. 1995, ApJ, 450, 253

Huang, Y. F., \& Lu, T. 1997, A\&A, 325, 189

Kettner, Ch., Weber, F., Weigel, M. K., \& Glendenning, N. K. 1995, Phys. Rev. D, 51, 1440

Manchester, R. N., \& Tayor, J. H. 1981, AJ, 86, 1953

Phukon, T. C. 2000, Phys. Rev. D, 62, 023002

Thompson, C., \& Duncan, R. C. 1993, ApJ, 408, 194

Usov, V. V. 1998, Phys. Rev. Lett., 80, 230

Usov, V. V. 2001a, invited talk at the Conference on Compact Stars in the QCD Phase Diagram, Copenhagen, Aug. 15-18 [arXiv:astro-ph/0111442]

Usov, V. V. 2001b, ApJ, 550, L179

Xu, R. X., Zhang, B., \& Qiao, G. J. 2001, Astropart. Phys., 15, 101 